\title{
Remission of concomitant Henoch-Schöenlein purpura and Sydenham chorea after intravenous corticosteroids
}

\author{
Carlos Zúñiga, Sergio Díaz, Ángeles Fariña, Federico M icheli \\ Parkinson's Disease and Movement Disorders Unit, Hospital De Clinicas "José De San Martin", Buenos Aires, Argentina
}

Address for correspondence:

Dr. Carlos Zúñiga,

Av. Tepeyac 1287, Col. Chapalita,

C.P. 45040, Zapopan, México.

E-mail: c.zuniga.ramirez@gmail.com

DOI: $10.4103 / 0028-3886.48819$

\begin{abstract}
Abstrat
We report a young girl who developed Henoch-Schöenlein purpura at the age of 11 years. Two weeks later she developed severe asymmetric choreic movements and behavioral disturbances. Sydenham's chorea was diagnosed based on the laboratory evidence and she was given intravenous methylprednisolone for five consecutive days. Both behavioral and movement disorder rapidly resolved. She was asymptomatic at three years of follow-up. The rapid resolution of choreic movements and behavioral disturbances in our patients suggests, intravenous corticosteroids may be an option in the treatment of Sydenham's chorea, more so when the movements are disabling.
\end{abstract}

Key words: Henoch-schöenlein purpura, rheumatic fever, sydenham's chorea intravenous corticosteroids

\section{Introduction}

Sydenham's chorea (SC) is one of the manifestations of rheumatic fever, a complication of the infection caused by a $\beta$-hemolytic Streptococcus Group A, a condition most frequently seen in developing countries. Treatment of choreic symptoms in SC with corticosteroids has long been known, ${ }^{[1]}$ However, most of the experience comes from uncontrolled trials and anecdotal reports. Recently, a randomized controlled trial with oral prednisone in children with SC showed promising results. ${ }^{[2]}$ Trial with corticosteroids have been conducted before for the treatment of other types of choreas, presupposing the existence of common pathophysiologic mechanisms and mode of action. ${ }^{[3]}$

Henoch-Schöenlein purpura (HSP) is the most frequent vasculitis in children related to streptococcal infection which is thought to be triggered by an autoimmune mechanism. ${ }^{[4]}$ We describe a girl with concomitant HSP and SC secondary to a streptococcal infection, who responded dramatically to intravenous (IV) corticosteroid.

\section{Case Report}

A girl aged 11-years developed upper respiratory tract infection in April 2004, however no medical attention was sought then. A few days later she developed purpuric rash on both the lower limbs and abdomen accompanied by abdominal pain and arthralgia. Initial evaluation showed elevated white blood cell count, microscopic haematuria and proteinuria. A diagnosis of HSP was made. Two weeks later, she developed behavioral changes, poor scholastic performance and involuntary movements of the right side of her body, which later became generalized over the next one week. She was examined in our department one month later. The patient was found to be inattentive, impulsive, anxious, irritable, reluctant to answer questions, prone to crying, and exhibited several ideomotor and motor dyspraxias, generalized choreic movements, mainly on the right side, which made both gait and performance of manual tasks difficult. In addition, she had mild dysarthria. Neuropsychological tests including WISC III, and NEPSY showed a normal intelligence quotient. 
However, difficulties were detected in recovery of information and severe dyspraxia was noted, suggestive of fronto-limbic dysfunction. Laboratory tests showed high levels of anti-streptolysins (425 Todd U), an elevated Streptozyme agglutination titer $(>200)$ and a $C$ reactive protein value of $4 \mathrm{mg} / \mathrm{L}$. Other laboratory studies were normal, and so were brain magnetic resonance imaging (MRI), an electrocardiogram and a two-dimensional echocardiogram. She was given IV methylprednisolone $(25 \mathrm{mg} / \mathrm{kg} /$ day) for five consecutive days. During IV corticosteroid therapy, marked and prompt improvement was observed in behavioral disorders, and the choreic movements almost completely remitted. She was started on prophylactic benzatinic penicillin. After discharge, the patient was periodically examined. No relapse of symptoms and she has been asymptomatic.

\section{Discussion}

Sydenham's chorea is still a frequent entity in developing countries. As much as $68 \%$ of the patients with a diagnosis of chorea who present to medical centers have this condition. Moreover, this shows that the choreic phenomenon per se, whether active or in remission, accompanies rheumatic fever in almost a third of the cases registered. ${ }^{[5]}$

Corticosteroid therapy has been considered as first-line treatment in different studies. Oral prednisone is the most widely used and successfully controls the movement disorder, with a very low relapse or complication rate. ${ }^{[6]}$ In general, clinical improvement is seen within the first 48 hours, and a remission of the motor and neuropsychiatric manifestations is seen within seven to 12 days after the initiation of corticosteroid therapy. ${ }^{[7]}$ Intravenous methylprednisolone pulses for five consecutive days have been used in chronic cases refractory to conventional therapies with good results. Complete remission observed in two of the five subjects treated with this treatment and in the rest a significant improvement was observed. ${ }^{[8]}$ In recent study four children with acute SC were treated with IV methylprednisolone pulses. A significant improvement was noted during the first month of treatment and this effect lasted for a year. ${ }^{[9]}$ Our patient showed an excellent response to IV steroid therapy, and remission was faster and more complete in comparison with the cases previously described.

Cognitive, neurological and psychiatric manifestations associated with this type of condition are diverse. It is thought that they are secondary to an antibodymediated dysfunction of the basal ganglia. ${ }^{[10]}$ Paediatric autoimmune neuropsychiatric disorder associated with Streptococcal infection (PANDAS) is characterized by neuropsychiatric disorders accompanied by tics and chorea-like movements, which are evoked by means of complex postures remitting when the patient is at rest. However, this syndrome is not considered a forme frustre of SC, but may be an overlap syndrome. ${ }^{[1]]} \mathrm{To}$ date, no definite clinical criteria are evolved for these two entities. ${ }^{[12]}$ It seems that both the pathologies are clinically and immunologically different. Our patient exhibited an improvement of her neuropsychiatric symptoms after corticosteroid therapy. It is believed that these manifestations are mediated by autoimmune mechanisms, with a production of antibodies directed against the basal ganglia. This can be seen as hypermetabolism of the striatum observed in single photon emission computed tomography (SPECT) scans. ${ }^{[13]}$ Previous findings in MRI volumetric studies suggest the presence of a local inflammatory process in the basal ganglia in PANDAS and in SC. ${ }^{[14,15]}$ Although PANDAS and SC are two distinct entities, both may be related to the same pathogen. Besides that, neuropsychiatric conditions can also be seen in the later, including obsessive compulsive disorder (OCD) and attention deficit hyperactivity disorder (ADHD), being more frequent in the chronic forms of the disease. ${ }^{[16]}$

HSP is the most common form of vasculitis in childhood. Although diagnostic criteria had been established since early nineties, there may be still some discordance among them. A recent study, including 68 patients with HSP, found that only $17 \%$ of this population fulfilled both sets of criteria, showing that the concordance between these two systems of classification is low. ${ }^{[17]}$ The association of HSP and rheumatic fever has been previously reported. Cardiac involvement was the most important hallmark among these reports. In general, patients were treated with prednisone, presenting a significant clinical improvement with echocardiographic follow-up that showed considerable amelioration of their previous condition..$^{[4,18,19]}$ As regards our patient, we did not find clinical or echocardiographic data indicating valve involvement or a history of arthralgia or renal disease.

Our case showed an optimal response to IV methylprednisolone, and for more than three years no relapse has been observed. However, in a study which assessed 24 subjects, SC relapse was observed in 10 of the total number of patients examined. This condition occurred within the first three months and 10 years after the onset of the disease: eight of them within the first year and a half. So far, this is the highest relapse rate published to date (42\%). ${ }^{[20]}$ Other treatment modalities described for motor signs and symptoms include the use of carbamazepine and valproate, both with a similar response and a relapse rate below 20\%. ${ }^{[21]}$ Another study showed slightly higher results in subjects treated with valproate and carbamazepine as compared to those 
treated with haloperidol. ${ }^{[22]}$

This is the first case reported depicting the coexistence of SC and HSP treated with IV corticosteroids, showing a remarkable response without relapse as to any of the two conditions for more than three years. Experience with this treatment modality among patients with SC had been scarce. Nevertheless, the responses obtained in this particular case, as well as in those reported by Texeira et al., suggest that IV corticosteroids should be considered as a possible therapeutic alternative in SC, especially in cases with disabling disease or in those in whom acute response from treatment is desired. Further studies evaluating the safety and effectiveness of IV corticosteroids in SC are needed.

\section{References}

1. Green LN. Corticocorticosteroids in the treatment of Sydenham's chorea. Arch Neurol 1978;35:53-4.

2. Paz JA, Silva CA, Marques-Dias MJ. Randomized double-blind study with prednisone in Sydenham's chorea. Pediatr Neurol 2006;34:264-9.

3. Brown WT, Sanberg PR, McGeer PL. Corticocorticosteroids and chorea. Arch Neurol 1979;36:452-3.

4. Eisenstein EM, Navon-Elkan P. Acute rheumatic fever associated with Henoch-Schonlein purpura: Report of three cases and review of the literature. Acta Paediatr 2002;91:1265-7.

5. Cardoso F, Eduardo C, Silva AP, Mota CC. Chorea in fifty consecutive patients with rheumatic fever. Move Disord 1997;12:701-3.

6. Ghram N, Allani C, Oudali B, Fitouri Z, Ben Becher S. Sydenham's chorea in children. Arch Pediatr 1999;6:1048-52.

7. Barash J, Margalith D, Matitiau A. Corticocorticosteroid treatment in patients with Sydenham's chorea. Pediatr Neurol 2005;32:205-7.

8. Cardoso F, Maia D, Cunningham MC, Valenca G. Treatment of Sydenham chorea with corticocorticosteroids. Mov Disord 2003;18:1374-7.

9. Teixeira AL, Maia DP, Cardoso F. Treatment of acute Sydenham's chorea with methyl-prednisolone pulse-therapy. Parkinson Relat Disord 2005; $11: 327-30$
10. Pineda M. Afectación neurológica en las enfermedades reumáticas y vasculitis de la infancia. Rev Neurol 2002;35:290-6.

11. Pavone P, Parano E, Rizzo R, Trifiletti RR. Autoimmune neuropsychiatric disorders associated with streptococcal infections: Sydenham chorea, PANDAS, and PANDAS variants. J Child Neurol 2006;21:727-36.

12. Swedo SE, Leonard HL, Rapoport JL. The Pediatric Autoimmune Neuropsychiatric Disorders Associated With Streptococcal Infection (PANDAS) Subgroup: Separating Fact From Fiction. Pediatrics 2004:113:907-11.

13. Lee PH, Nam HS, Lee KY, Lee BI, Lee JD. Serial brain SPECT images in a case of Sydenham chorea. Arch Neurol 1999;56:237-40.

14. Giedd JN, Rapoport JL, Garvey MA, Perlmutter S, Swedo SE. MRI Assessment of Children with obsessive-compulsive disorder or tics associated with streptococcal infection. Am J Psychiatry 2000;157:281-3.

15. Giedd JN, Rapoport JL, Kruesi MJ, Parker C, Schapiro MB, Allen AJ, et al. Sydenham's chorea: Magnetic resonance imaging of the basal ganglia. Neurology 1995;45:2199-202.

16. Maia DP, Teixeira AL, Cunningham MCQ, Cardoso F. Obsessive compulsive behavior, hyperactivity and attention deficit disorder in Sydenham chorea. Neurology 2005;64:1799-1801.

17. Murali NS, George R, Chandi SM, Chandi SM, Jacob M, Jeyaseelan L, et al. Problems of classification of Henoch Schonlein purpura: An Indian perspective. Clin Exp Dermatol 2002;27:260-3.

18. Torres J, de Oliveira S, de Almeida R, Pelajo C, Sztajnbok F, Rodrigues MC. Acute rheumatic fever associated with Henoch-Schonlein purpura: Case report and review of the literature. Acta Reumatol Port 2007;32:377-80

19. Gulati T, Kumar P, Dewan V, Anand VK. Henoch schonlein purpura with rheumatic carditis. Indian J Pediatr 2004;71:371-2.

20. Korn-Lubetzki I, Brand A, Steiner I. Recurrence of Sydenham chorea implications for pathogenesis. Arch Neurol 2004;61:1261-4.

21. Genel F, Arslanoglu S, Uran N, Saylan B. Sydenham's chorea: Clinical findings and comparison of the efficacies of sodium valproate and carbamazepine regimens. Brain Dev 2002;24:73-6.

22. Peña J, Mora E, Cardozo J, Molina O, Montiel C. Comparison of the efficacy of carbamazepine, haloperidol and valproic acid in the treatment of children with Sydenham's chorea. Arq Neuropsiquiatr $2002 ; 60: 374-7$

Accepted on 08-02-2009

Source of Support: Nil, Conflict of Interest: None declared. 